\title{
Article
}

\section{Ion Limited Charging of Nanoparticle Thin Films}

Timo Laaksonen, Virginia Ruiz, Peter Liljeroth, and Bernadette M. Quinn

J. Phys. Chem. C, 2008, 112 (40), 15637-15642 • DOI: 10.1021/jp8048108 • Publication Date (Web): 12 September 2008

Downloaded from http://pubs.acs.org on January 14, 2009

\section{More About This Article}

Additional resources and features associated with this article are available within the HTML version:

- Supporting Information

- Access to high resolution figures

- $\quad$ Links to articles and content related to this article

- Copyright permission to reproduce figures and/or text from this article

\section{View the Full Text HTML}




\title{
Ion Limited Charging of Nanoparticle Thin Films
}

\author{
Timo Laaksonen, ${ }^{\dagger}$ Virginia Ruiz,,$\ngtr$ Peter Liljeroth, ${ }^{\S}$ and Bernadette M. Quinn*,\| \\ Division of Pharmaceutical Technology, Faculty of Pharmacy, University of Helsinki, P.O. Box 56, FIN-00014 \\ University of Helsinki, Finland, Department of Engineering Physics, Helsinki University of Technology, \\ P.O. Box 5100, FIN-02015 HUT, Finland, Condensed Matter and Interfaces, Debye Institute for Nanomaterials \\ Science, University of Utrecht, P.O. Box 80000, 3508 TA Utrecht, The Netherlands, and Department of \\ Chemistry, Helsinki University of Technology, P.O. Box 6100, FIN-02015 HUT, Finland
}

Received: May 31, 2008; Revised Manuscript Received: July 16, 2008

\begin{abstract}
This article reports on the charging behavior of thin films of alkanethiol protected gold nanoparticles, socalled monolayer protected clusters (MPCs), immersed in aqueous solution. We demonstrate that the oxidation of the MPCs is controlled by the transfer of counter-ions across the aqueous/MPC film interface. A model is developed to describe this and its predictions compared with experiment. Langmuir-Schaefer MPC thin films were transferred to a glassy carbon electrode and film charging behavior investigated in a series of aqueous solutions comprised of different base electrolyte anions. The dependence of peak current on film thickness and peak position on anion lipophilicity for MPC oxidative charging can all be accounted for with the ion-transfer limited model. The impact of ion partitioning into the film at equilibrium is also discussed and the effect of the aqueous phase cation is theoretically considered. In addition, the effect of co-transported water with hydrophilic ions transferring into the film on film charging is rationalized.
\end{abstract}

\section{Introduction}

Gold nanoparticles coated by an alkanethiol monolayer, socalled monolayer protected clusters (MPCs), are the focus of intense study due to their interesting optical and electronic properties. $^{1,2}$ Dispersed MPCs have been considered both as diffusing nanoelectrodes and as conventional redox molecules. ${ }^{2}$ Due to a combination of nanometer core size and the subattofarad capacitance associated with the protecting monolayer, electron transfer to the metal core is discrete at room temperature. ${ }^{2}$ In electrochemical experiments, voltammetry of solutions of dispersed MPCs yields a series of regularly spaced current peaks where each peak corresponds to single electron/hole charging of the metal core. ${ }^{3-6}$ The voltage interval between peaks, $\Delta E$, is determined by the MPC capacitance. ${ }^{2}$ The charging response is significantly different when the particles are immobilized on a substrate and immersed in aqueous electrolyte. ${ }^{7-10}$ For instance, the oxidative charging of hydrophobic MPC thin films coated on electrodes and immersed in aqueous solution depends on the nature and concentration of the aqueous electrolyte anion while reductive charging has not been observed. ${ }^{9}$ This latter effect has yet to be rationalized. The anion influence on the onset potential for film oxidation has been studied in detail. ${ }^{7-9,11,12}$ The onset potential for the oxidation in voltammetric measurements is dependent on the nature of the anion and shifts to more negative potentials with increasing anion hydrophobicity in the following order: $\mathrm{NO}_{3}{ }^{-}$ $<\mathrm{BF}_{4}{ }^{-}<\mathrm{ClO}_{4}{ }^{-}<\mathrm{PF}_{6}{ }^{-}$. 9 This apparent anion rectifying effect has been interpreted in terms of ion association where the oxidized MPC ion pairs with the electrolyte anion. ${ }^{8-10,12}$ The shift in onset potential would then be due to the differing iki.fi.

* To whom correspondence should be addressed. E-mail: bquinn@

$\dagger$ University of Helsinki.

¥ Department of Engineering Physics, Helsinki University of Technology.

\& University of Utrecht.

" Department of Chemistry, Helsinki University of Technology.
$\mathrm{MPC}^{+}-$anion association constants, with more hydrophobic ions binding more strongly. However, ion association cannot explain why comparable ion-induced rectification is not seen when the films are immersed in lower permittivity solvents such as dichloromethane. ${ }^{13,14}$ The ion-pairing based interpretation does not take into account the solvation barrier for the transfer of charge compensating counter-ions into the film when the particles are oxidized. ${ }^{15,16}$ As the film and aqueous phases are mutually immiscible, there is an energy cost to transfer an ion from one phase to the other that is dependent on the solvation energy of the ion in the film versus the aqueous phase. ${ }^{17}$ This implies that there are two polarizable interfaces and the applied potential is distributed across both of them. ${ }^{15,16}$ The potential distribution across the electrode/film and film/water interfaces is illustrated in Figure 1a. Due to the electroneutrality condition, electron transfer (ET) at the electrode/film interface is coupled to ion transfer (IT) across the aqueous/film interface. ET and IT occur simultaneously and cannot be separated. ${ }^{15,16}$ In the simple case, this means that oxidation of the MPCs must be coupled to anion injection from the aqueous phase into the film (Figure 1b). ${ }^{11}$ We will also consider the general case where the aqueous base electrolyte can partition into the film and oxidation of the MPCs is coupled to either anion injection or cation ejection (Figure 1c). Previously, we have demonstrated how differences in ion solvation in the film versus the electrolyte phase can control the apparent redox properties of MPC thin films. ${ }^{11}$ This ion limited model does not require any a priori interaction between the base electrolyte anion and the oxidized MPC. Here we give a full description of the model and compare the model predictions with experimental data both from the literature and on thin films reported here.

To compare model predictions with experimental data on films of controlled thickness, the Langmuir-Schaefer (LS) technique was used to form MPC multilayer films. This method enables precise control over film thickness, roughness, and MPC concentration in the film in contrast to drop-casting or spin- 

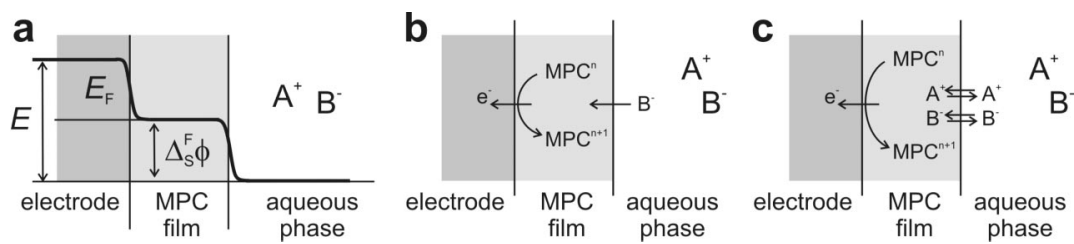

Figure 1. (a) Schematic of the potential distribution across the polarizable electrode/film and film/water interfaces. (b) Illustration of coupled MPC oxidation and anion injection for the charging of hydrophobic MPC films in aqueous solution. (c) Schematic of the general case where the aqueous base electrolyte can partition into the MPC film and MPC oxidative charging is coupled to either cation ejection or anion injection process.

coating methods. Voltammetry of the transferred films of differing thicknesses was undertaken in different aqueous electrolyte anions. We demonstrate that the model can fully account for all experimental observations for oxidative charging of MPC films. The influence of ion partitioning on oxidative charging was also theoretically considered, and predictions were compared with literature data.

\section{Experimental Section}

Preparation and Characterization of MPCs. Hexanethiolate MPCs (C6 MPCs) were synthesized according to a literature procedure known to yield particles of small core diameter. ${ }^{3,5}$ The synthesis yields particles with a dominant population of monodisperse cores of average radius of $0.81 \mathrm{~nm}$, estimated from electrochemical measurements using the simple concentric sphere capacitor model as described previously. ${ }^{2}$ The particles were assigned $\mathrm{Au}_{147}$ based on previous reports. ${ }^{2,4,5}$

Langmuir Deposition. The particles were spread from very dilute chloroform solutions $\left(0.2 \mathrm{mg} \mathrm{mL}^{-1}\right)$ onto a pure water (MQ, Millipore) subphase in a KSV minitrough (KSV Instruments, Finland) thermostatted at $20{ }^{\circ} \mathrm{C}$. The spreading solvent was allowed to evaporate for at least $1 \mathrm{~h}$ prior to compression. The compression rate used throughout was $5 \mathrm{~mm} \mathrm{~min}^{-1}$. A typical Langmuir isotherm obtained for a monolayer of MPCs at the air/water interface is given in the Supporting Information. The monolayers were transferred at a surface pressure just before the collapse point by the LS method, i.e., horizontal dipping of the substrate. A $5 \mathrm{~mm}$-diameter glassy carbon (GC) electrode was brought down horizontally by hand to touch the monolayer and lifted carefully after a contact time of a few seconds. This was then repeated to transfer a given number of monolayers. Films investigated here consisted of 15-35 LS monolayers. Thus, assuming that the thickness of a monolayer is that of an MPC $\left(l_{\mathrm{MPC}}=3.16 \mathrm{~nm}\right)$, film thicknesses ranged between ca. 50 and $110 \mathrm{~nm}$. The area occupied per MPC, $A_{\mathrm{MPC}}$, was estimated from TEM images of MPC monolayers transferred analogously onto carbon coated grids at the same surface pressures (Supporting Information).

Electrochemical Measurements. Cyclic voltammograms (CVs) and differential pulse voltammograms (DPVs) of the MPC films on GC were conducted in aqueous solutions containing various base electrolytes using a PGSTAT 100 potentiostat (Eco Chemie B.V., The Netherlands). A threeelectrode arrangement was used with a silver wire and a Pt coil as quasireference and counter electrodes, respectively. Prior to use, the GC was polished using $0.05 \mu \mathrm{m}$ alumina slurries, rinsed copiously with MQ water, and sonicated in MQ water. The base electrolytes used, $\mathrm{NH}_{4} \mathrm{PF}_{6}, \mathrm{NH}_{4} \mathrm{ClO}_{4}, \mathrm{NH}_{4} \mathrm{BF}_{4}$, and $\mathrm{NH}_{4} \mathrm{NO}_{3}$, were of the highest available commercial purity and were used as received.

The charging behavior of the film modified GC electrode in aqueous media was investigated by cyclic voltammetry. Several test LS films were initially prepared, composed of between 15 and 35 monolayers to investigate the influence of monolayer surface pressure on film charging. It was found that the MPC charging peaks were better defined as surface pressure, and hence, the number of MPCs per unit area increased. Consequently, all films were transferred at pressures just before the collapse point $\left(14-16 \mathrm{mN} \mathrm{m}^{-1}\right)$.

\section{Results and Discussion}

In the following sections, we first outline the theoretical treatment for the coupling of ion and electron transfers for the MPC film modified electrode immersed in aqueous solution and show how the experimental data obtained at model LangmuirSchaefer thin films agrees well with theoretical predictions. The influence of all ions present in the aqueous phase on the IT-ET coupling is then considered with reference to data in the literature.

Theory of Coupled Ion and Electron Transfer. For a thin hydrophobic redox film cast on an electrode surface immersed in aqueous base electrolyte solution, the measured potential $E$ is a sum of the potential drops across the electrode/film $E_{\mathrm{F}}$ and film/solution $\Delta_{\mathrm{s}}^{\mathrm{F}} \phi$ interfaces (Figure 1a). ${ }^{15,16}$

$$
E=E_{\mathrm{F}}+\Delta_{\mathrm{s}}^{\mathrm{F}} \phi
$$

where $\mathrm{F}$ and $\mathrm{s}$ denote the film and solution phases, respectively. As the film and aqueous phases are mutually immiscible, there is a considerable solvation barrier for ions to transfer from one phase to the other. ${ }^{17}$ Due to this barrier, the potential drop across the film/solution interface has to be included in the expression for $E$ in eq 1. Due to the electroneutrality condition, electron transfer at the electrode/film interface and ion transfer at the film/solution interface are coupled and cannot be separated..$^{15,16}$ Thus, the oxidation of the MPC must be accompanied by the injection/expulsion of a charge compensating counterion across the film/solution interface. ${ }^{15,16}$ The overall cell reaction can be either of the following:

$$
\begin{aligned}
& \mathrm{MPC}^{n}+\mathrm{B}_{\mathrm{s}}^{-} \rightleftharpoons \mathrm{MPC}^{n+1}+\mathrm{B}_{\mathrm{F}}^{-}+\mathrm{e}^{-} \\
& \mathrm{MPC}^{n}+\mathrm{A}_{\mathrm{F}}^{+} \rightleftharpoons \mathrm{MPC}^{n+1}+\mathrm{A}_{\mathrm{s}}^{+}+\mathrm{e}^{-}
\end{aligned}
$$

The charge compensating ion transfer reaction can be either $\mathrm{B}^{-}$transfer from the aqueous phase to the film phase or $\mathrm{A}^{+}$ transferring in the opposite direction. In the following sections, we model the voltammetric response when the electron transfer is coupled to both anion injection and cation ejection, reactions 2a,b. For simplicity, we only consider the first oxidation peak where $n=1$, as this is a measure of the onset potential. The theoretical treatment is general and is equally valid for higher oxidation states. We assume that the volume of the aqueous phase is much larger than that of the MPC film, $r=V_{\mathrm{F}} / V_{\mathrm{s}} \rightarrow$ 0 . This means that the concentrations in the aqueous phase remain constant and equal to the bulk concentrations of the salt, $c_{\mathrm{AB}}^{*}$.

$$
c_{\mathrm{A}}^{\mathrm{s}}=c_{\mathrm{B}}^{\mathrm{s}}=c_{\mathrm{AB}}^{*}
$$

We can write a Nernst equation for the ion transfer reaction between the aqueous phase and MPC film for all the ions ${ }^{17}$ 


$$
\begin{aligned}
& \Delta_{\mathrm{s}}^{\mathrm{F}} \phi=\Delta_{\mathrm{s}}^{\mathrm{F}} \phi_{\mathrm{A}}^{0^{\prime}}+\frac{R T}{F} \ln \frac{c_{\mathrm{AB}}^{*}}{c_{\mathrm{A}}^{\mathrm{F}}} \\
& \Delta_{\mathrm{s}}^{\mathrm{F}} \phi=\Delta_{\mathrm{s}}^{\mathrm{F}} \phi_{\mathrm{B}}^{0^{\prime}}+\frac{R T}{F} \ln \frac{c_{\mathrm{B}}^{\mathrm{F}}}{c_{\mathrm{AB}}^{*}}
\end{aligned}
$$

where $\Delta_{\mathrm{s}}^{\mathrm{F}} \phi_{i}^{0^{\prime}}$ is the standard transfer potential of ion $i$ between the aqueous phase and the MPC film. Similarly, we can write the Nernst equation for the electron transfer between the electrode and the MPCs in the film

$$
E_{\mathrm{F}}=E_{\mathrm{MPC}}^{0^{\prime}}+\frac{R T}{F} \ln \frac{c_{\mathrm{MPC}+}}{c_{\mathrm{MPC} 0}}
$$

where $E_{\mathrm{MPC}}^{0^{\prime}}$ is the standard redox potential of the first MPC oxidation. The total concentration of the MPCs, $c_{\mathrm{MPC}}^{*}$, remains constant

$$
c_{\mathrm{MPC}+}+c_{\mathrm{MPC} 0}=c_{\mathrm{MPC}}^{*}
$$

Using eqs 5 and 6 gives for the MPC concentrations

$$
\begin{gathered}
c_{\mathrm{MPC} 0}=\frac{c_{\mathrm{MPC}}^{*}}{1+\theta_{\mathrm{MPC}}} \\
c_{\mathrm{MPC}+}=\frac{\theta_{\mathrm{MPC}} c_{\mathrm{MPC}}^{*}}{1+\theta_{\mathrm{MPC}}}
\end{gathered}
$$

where $\theta_{\mathrm{MPC}}=\exp \left(F / R T\left(E_{\mathrm{F}}-E_{\mathrm{MPC}}^{0^{\prime}}\right)\right)$. Electroneutrality condition for the MPC film is

$$
c_{\mathrm{MPC}+}+c_{\mathrm{A}}^{\mathrm{F}}-c_{\mathrm{B}}^{\mathrm{F}}=0
$$

Using eqs $3,4 \mathrm{a}$, and $7 \mathrm{a}$ gives

$$
c_{\mathrm{AB}}^{*}\left(1 / \theta_{\mathrm{A}}-\theta_{\mathrm{B}}\right)+c_{\mathrm{MPC}}^{*} \theta_{\mathrm{MPC}} /\left(1+\theta_{\mathrm{MPC}}\right)=0
$$

where $\theta_{i}=\exp \left(F / R T\left(\Delta_{\mathrm{s}}^{\mathrm{F}} \phi-\Delta_{\mathrm{s}}^{\mathrm{F}} \phi_{i}^{0^{\prime}}\right)\right)$ for $i=\mathrm{A}$, B.

To model the corresponding cyclic voltammetric response, we assume that the MPC film behaves as an ideal thin film, where diffusion and concentration gradients can be neglected..$^{18}$ The validity of the assumption is dependent on both the thickness of the film and the diffusion coefficient of the species inside the film. ${ }^{18}$ The electron diffusion coefficient is known to be as low as $10^{-8} \mathrm{~cm}^{2} / \mathrm{s},{ }^{19}$ which means that the onset of diffusion control would occur at a thickness of ca. 100-500 $\mathrm{nm}$, the same scale as has been seen experimentally. In the following, we only consider films thinner than this, where diffusion inside the film can be neglected. For an ideal thin film, the current can be defined as ${ }^{18}$

$$
i=F A l \frac{\mathrm{d} c_{\mathrm{MPC}+}}{\mathrm{d} t}
$$

where $A$ is the electrode area and $l$ is the film thickness. For a given applied potential, eqs 1 and 9 can be solved numerically to give $E_{\mathrm{F}}$ and $\Delta_{\mathrm{s}}^{\mathrm{F}} \phi$ at that potential. These can then be used to obtain the concentrations of all the species. Finally, the current can be calculated from eq 10 .

Voltammetric Response in the Case Where Only Anion Injection Is Relevant. As the film is initially assumed to be ion-free and the aqueous electrolyte is typically composed of a hydrophilic cation (e.g., $\mathrm{NH}_{4}{ }^{+}$) and a relatively hydrophobic anion (e.g., $\mathrm{PF}_{6}{ }^{-}$), a typical special case is that the anion transfer from the aqueous phase to the film phase is the charge compensating ion transfer reaction..$^{7-12}$ In this case, the elec- troneutrality condition is simplified to $c_{\mathrm{B}}^{\mathrm{F}}=c_{\mathrm{MPC}+}$. Using this simplification and eqs $1,4 \mathrm{a}-6$ yields the following expression for $c_{\mathrm{MPC}+}$

$$
c_{\mathrm{MPC}^{+}}=\frac{c_{\mathrm{AB}}^{*} \theta}{2}\left[-1+\left(1+4 \frac{c_{\mathrm{MPC}}^{*}}{c_{\mathrm{AB}}^{*} \theta}\right)^{1 / 2}\right]
$$

where

$$
\theta=\exp \left[\frac{F}{R T}\left(E-E_{\mathrm{MPC}}^{0^{\prime}}-\Delta_{\mathrm{s}}^{\mathrm{F}} \phi_{\mathrm{B}}^{0^{\prime}}\right)\right]
$$

Inserting eq 11 into eq 10, we get the following expression for the voltammetric current

$$
\begin{aligned}
& i=\frac{F^{2} A l v}{R T}\left(\frac{c_{\mathrm{AB}}^{*} \theta}{2}\left[-1+\left(1+4 \frac{c_{\mathrm{MPC}}^{*}}{c_{\mathrm{AB}}^{*} \theta}\right)^{1 / 2}\right]\right. \\
&\left.c_{\mathrm{MPC}}^{0}\left[1+4 \frac{c_{\mathrm{MPC}}^{*}}{c_{\mathrm{AB}}^{*} \theta}\right]^{-1 / 2}\right)
\end{aligned}
$$

where $v$ is the sweep rate. Equation 13 can then be solved for the peak current $i_{\mathrm{p}}$ and potential $E_{\mathrm{p}}$

$$
\begin{gathered}
i_{\mathrm{p}}=\frac{F^{2} A l v c_{\mathrm{MPC}}^{*}}{5.83 R T} \\
E_{\mathrm{p}}=E^{0^{\prime}}+\Delta_{\mathrm{s}}^{\mathrm{F}} \phi_{\mathrm{B}}^{0^{\prime}}-\frac{R T}{F} \ln c_{\mathrm{AB}}^{*}+\frac{R T}{F} \ln 0.83 c_{\mathrm{MPC}}^{*}
\end{gathered}
$$

The predictions of this simple model can be summarized as follows: from eq 14 peak current is linearly dependent on the MPC film concentration, film thickness, and scan rate while eq 15 shows that the peak position has a logarithmic dependence on both base electrolyte anion and MPC concentrations. The influence of the anion lipophilicity on $E_{\mathrm{p}}$ is accounted for in the $\Delta_{\mathrm{S}}^{\mathrm{F}} \phi_{\mathrm{B}}^{0^{\prime}}$ term and shifts negative as lipophilicity increases, i.e., $\Delta_{\mathrm{s}}^{\mathrm{F}} \phi_{\mathrm{B}}^{0^{\prime}}$ decreases.

Comparing Theory and Experiment. We will first consider base electrolytes with a very hydrophilic cation, and hence, we only need to consider anion transfer as the charge compensating mechanism. To compare the predictions of eqs 14 and 15 with experimental data, we need precise control of the film thickness. This can be achieved by using the Langmuir-Schaefer method to transfer MPC monolayers to a GC electrode where the thickness of the film is simply the diameter of MPC times the number of transferred monolayers. GC electrodes modified with films of controlled thickness were then characterized by voltammetry in aqueous solutions of various base electrolytes. Representative CVs of such a 25-monolayer MPC film transferred on a GC electrode in an aqueous solution of $0.1 \mathrm{M}$ $\mathrm{NH}_{4} \mathrm{PF}_{6}$ are shown in Figure 2a. The negligible peak separation between forward and reverse scans for all the scan rates confirms that the 25-monolayer MPC film is behaving as an ideal thin film. As mass transport inside the film is not limiting the charging response, the expressions outlined above can be used to quantify charging. Using eq 14 , the MPC concentration in the transferred film was determined from the slope of the linear plot of peak current for the first charging peak versus scan rate. Each layer of transferred MPCs was assumed to be $3.16 \mathrm{~nm}$ thick in these calculations. The inset of Figure $2 \mathrm{a}$ shows experimental values along with the linear fit for films in aqueous solutions of $0.1 \mathrm{M} \mathrm{NH}_{4} \mathrm{PF}_{6}$ electrolyte solution. Comparable linear dependences for $i_{\mathrm{P}}$ versus $v$ with roughly identical slopes were obtained in $\mathrm{NH}_{4} \mathrm{ClO}_{4}, \mathrm{NH}_{4} \mathrm{BF}_{4}$, and $\mathrm{NH}_{4} \mathrm{NO}_{3}$ solutions, from which $c_{\text {MPC }}^{*}$ was calculated for each electrolyte. Table 1 summarizes the average $c_{\text {MPC }}^{*}$ values and corresponding standard 
TABLE 1: Experimentally Determined Values for the MPC Film Concentration, $E_{\mathrm{p}}$ and $\Delta_{\mathrm{s}}^{\mathrm{F}} \phi_{\mathrm{B}}^{0^{\prime}}+E^{0^{\prime}}$ Obtained in the Presence of Different Aqueous Phase Electrolytes

\begin{tabular}{lrccc}
\hline & \multicolumn{1}{c}{$\mathrm{NH}_{4} \mathrm{PF}_{6}$} & $\mathrm{NH}_{4} \mathrm{ClO}_{4}$ & $\mathrm{NH}_{4} \mathrm{BF}_{4}$ & $\mathrm{NH}_{4} \mathrm{NO}_{3}$ \\
\hline$c_{\mathrm{MPC}}^{*}(\mathrm{M})$ & $0.026 \pm 0.002$ & $0.026 \pm 0.002$ & $0.027 \pm 0.002$ & $0.027 \pm 0.002$ \\
$E_{\mathrm{p}}(\mathrm{V})$ vs QRE & $-0.062 \pm 0.006$ & $0.050 \pm 0.010$ & $0.113 \pm 0.015$ & $0.234 \pm 0.008$ \\
$\Delta_{\mathrm{s}}^{\mathrm{F}} \phi_{\mathrm{B}}^{0_{\mathrm{B}}}+E^{0^{\prime}}(\mathrm{V})$ & $-0.021 \pm 0.008$ & $0.089 \pm 0.013$ & $0.149 \pm 0.015$ & $0.271 \pm 0.010$
\end{tabular}

deviations determined from four different LS films transferred at the same surface pressure (two consisting of 25 MPC monolayers and the other two with 15 and 35 monolayers each).

Taking $l=l_{\mathrm{MPC}} \times n_{\text {layers }}$ and the average of the $c_{\mathrm{MPC}}^{*}$ values calculated in each electrolyte, the surface coverage can be estimated, $\Gamma_{\mathrm{MPC}}^{0}:(1.26 \pm 0.03) \times 10^{-10} \mathrm{~mol} \mathrm{~cm}^{-2}$ for a $15-$ monolayer film, $(2.09 \pm 0.04) \times 10^{-10} \mathrm{~mol} \mathrm{~cm}^{-2}$ for a 25 monolayer film, and $(2.99 \pm 0.08) \times 10^{-10} \mathrm{~mol} \mathrm{~cm}^{-2}$ for a 35 -monolayer film. Thus, $\Gamma_{\mathrm{MPC}}^{0}$ increases linearly with the number of monolayers transferred. The number of MPCs transferred per $\mathrm{cm}^{2}$ in a monolayer can be estimated from the TEM images $\left(8.70 \times 10^{-12} \mathrm{~mol} \mathrm{~cm}^{-2}\right)$ (Supporting Information). Dividing the electrochemically determined $\Gamma_{\mathrm{MPC}}^{0}$ for each film by this monolayer surface concentration gives the number of monolayers transferred. The values determined were 14.5, 24.0 , and 34.4, which are comparable to the actual number of transferred layers (number of dipping cycles). This again is a confirmation of the fidelity of monolayer transfer with the LS method and verifies the electrochemical method used to determine the surface concentration.

Previously, for drop-cast films, charging peak currents did not increase with film thickness for thicker films and tended to level off for very thick films. ${ }^{8}$ The concept of "electrochemically addressable layers" was introduced to explain this, and it was proposed that only the MPC layers in the vicinity of the film/ solution interface were active. ${ }^{8}$ Here we have demonstrated that the MPC surface concentration, and thus film thickness, is proportional to the peak current when the film is sufficiently thin to be considered as an ideal thin film and when the aqueous base electrolyte concentration is in excess. For thicker films, deviation will be observed as mass transfer inside the film becomes limiting. ${ }^{18}$ Thus, the concept of inactive layers does not have to be invoked to explain the current dependence on film thickness. It is simply a consequence of mass transfer in thin films, and the transition to the thick-film regime when electron transport in the film becomes limiting.

$\mathrm{CVs}$ for a 25-monolayer film in the presence of different base electrolyte anions are given in Figure 2b. The peak potential,
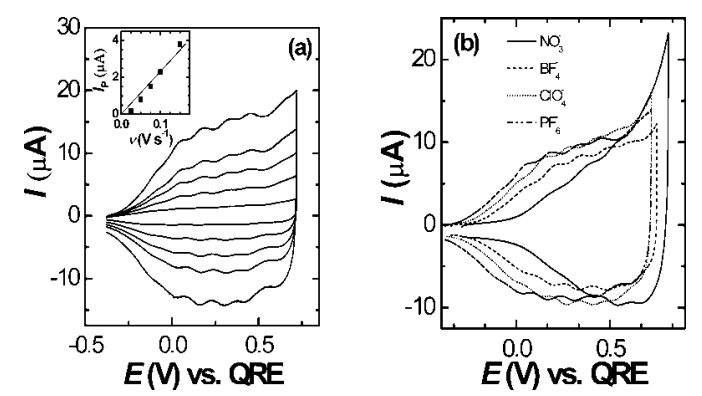

Figure 2. (a) Cyclic voltammograms of a 25-monolayer MPC film on GC immersed in $0.1 \mathrm{M} \mathrm{NH}_{4} \mathrm{PF}_{6}$ aqueous solution at different scan rates $\left(0.025,0.050,0.075,0.100\right.$, and $\left.0.150 \mathrm{~V} \mathrm{~s}^{-1}\right)$. Inset shows the linear dependence of the peak current for the first charging peak on the scan rate. The MPC concentration in the film was estimated from the slope. (b) Voltammograms of the same MPC film in $0.1 \mathrm{M}$ solutions of (from left to right): $\mathrm{NH}_{4} \mathrm{PF}_{6}, \mathrm{NH}_{4} \mathrm{ClO}_{4}, \mathrm{NH}_{4} \mathrm{BF}_{4}$, and $\mathrm{NH}_{4} \mathrm{NO}_{3}$. Scan rate: $0.1 \mathrm{~V} \mathrm{~s}^{-1}$.
$E_{\mathrm{p}}$, shifts in a cathodic direction when the base electrolyte anion is made progressively more hydrophobic, $\mathrm{PF}_{6}{ }^{-}>\mathrm{ClO}_{4}{ }^{-}>\mathrm{BF}_{4}{ }^{-}$ $>\mathrm{NO}_{3}{ }^{-}$, consistent with all previous reports. ${ }^{7-12}$ The magnitude of the shift is governed by the $\Delta_{\mathrm{s}}^{\mathrm{F}} \phi_{\mathrm{B}}^{0^{\prime}}$ term in eq 15 . Using the value of $c_{\mathrm{MPC}}^{*}, \Delta_{\mathrm{s}}^{\mathrm{F}} \phi_{\mathrm{B}}^{0^{\prime}}+E^{0^{\prime}}$ was calculated for each electrolyte anion using eq 15 for a given value of peak potential $\left(E_{\mathrm{p}}\right)$ for a given base electrolyte concentration. $E_{\mathrm{p}}$ values for the first oxidation were determined from $\mathrm{CV}$ s recorded at the slowest scan rate $\left(25 \mathrm{mV} \mathrm{s}^{-1}\right)$ run in each electrolyte. The calculated $\Delta_{\mathrm{s}}^{\mathrm{F}} \phi_{\mathrm{B}}^{0^{\prime}}+E^{0^{\prime}}$ for the four anions used are summarized in Table 1. The determination of the absolute value of $\Delta_{\mathrm{s}}^{\mathrm{F}} \phi_{\mathrm{B}}^{0^{\prime}}$ requires an independent estimate of $E^{0^{\prime}}$ and is nontrivial for our redox active films. ${ }^{15}$ However, as $E^{0^{\prime}}$ is independent of the anion used, the differences in $\Delta_{\mathrm{s}}^{\mathrm{F}} \phi_{\mathrm{B}}^{0^{\prime}}+E^{0^{\prime}}$ values obtained upon varying the anion can be ascribed to the anion dependent term $\Delta_{\mathrm{s}}^{\mathrm{F}} \phi_{\mathrm{B}}^{0^{\prime}}$. These differences are comparable to those determined using literature values for the standard ion transfer potentials between water and dichlorobenzene (DCB). ${ }^{17,20}$ The differences between ions are comparable to those reported for thick films indicating that the transfer energies are not influenced by the film thickness even for the case of the very thin films used here. ${ }^{11}$ Thus, the predictions of the model outlined above are consistent with experimental data.

Influence of the Aqueous Cation on Oxidative Charging. In the literature, the aqueous phase cation has also been reported to influence the position of $E_{\mathrm{p}}$. For a given anion, making the cation increasingly hydrophobic shifted $E_{\mathrm{p}}$ toward more positive potentials. ${ }^{9}$ We next consider how the coupled IT-ET model can account for this effect. As the film is assumed to be ionfree, it is counterintuitive to consider that cation transfer can also control the oxidative MPC charging as in reaction $2 \mathrm{~b}$ simply from a perceived lack of cations in the film phase. However, it is well established that contacting two immiscible phases results in a spontaneous redistribution of the ions depending on the relative lipophilicities of the ions dissolved in each phase. ${ }^{17} \mathrm{In}$ addition, as demonstrated by Kakiuchi, the volume ratio between the phases has a pronounced effect on ion partitioning when it approaches zero, $V_{\mathrm{F}} / V_{\mathrm{s}}=r \rightarrow 0 .{ }^{21}$ Lipophilic ions show enhanced dissolution in the smaller volume organic phase upon the addition of inorganic salts to the aqueous phase. ${ }^{21} \mathrm{We}$ now consider how the interplay between the relative lipophilicities of all ions added to solution can lead to a situation where the oxidative charging is controlled by cation ejection from the film without deliberately adding salt to the film phase. We consider a case where a 1:1 electrolyte $A^{+} B^{-}$is added to the aqueous phase and the film is initially assumed to be ion-free. CVs were simulated varying the lipophilicity of the aqueous ions through the $\Delta_{\mathrm{S}}^{\mathrm{F}} \phi_{\mathrm{B}}^{0^{\prime}}$ term. Literature values for ion transfer potentials across the w/DCB interface were used, ${ }^{17}$ and the $E^{0^{\prime}}$ value for MPC oxidation was taken as $0 \mathrm{~V}$. Concentrations of aqueous ionic species were varied from 0 to $0.1 \mathrm{M}$, and the MPC concentration in the film phase was taken to be $0.026 \mathrm{M}$ as measured for the LS thin films.

In this first instance, the effect of cation lipophilicity was considered for the case where only $\mathrm{A}^{+} \mathrm{B}^{-}$is present in the aqueous phase. The anion was assumed to be either $\mathrm{NO}_{3}{ }^{-}$ $\left(\Delta_{\mathrm{s}}^{\mathrm{F}} \phi_{\mathrm{NO}_{3}{ }^{-}}^{0^{\prime}}=0.489 \mathrm{~V} \text {, Figure } 3 \mathrm{a}\right)^{20}$ or $\mathrm{PF}_{6}{ }^{-}\left(\Delta_{\mathrm{s}}^{\mathrm{F}} \phi_{\mathrm{PF}_{6}}^{0^{\prime}}=0.165\right.$ $\mathrm{V}$, Figure $3 \mathrm{~b})^{20}$ and the cation lipophilicity was varied $-0.4 \mathrm{~V}$ $<\Delta_{\mathrm{s}}^{\mathrm{F}} \phi_{\mathrm{A}}^{0^{\prime}}<0.4 \mathrm{~V}$. The latter values are in the range reported for the transfer of sodium to long chain alkylammonium cations across the w/DCB interface..$^{17,20}$ The simulated CVs in the presence of $0.1 \mathrm{M}$ aqueous electrolyte $\mathrm{A}^{+} \mathrm{PF}_{6}{ }^{-}$given in Figure $3 \mathrm{~b}$ show that the peak potential is invariant with $\Delta_{\mathrm{S}}^{\mathrm{F}} \phi_{\mathrm{A}}^{0^{\prime}}$ until a given value $(0.1 \mathrm{~V})$ above which it increases linearly with 

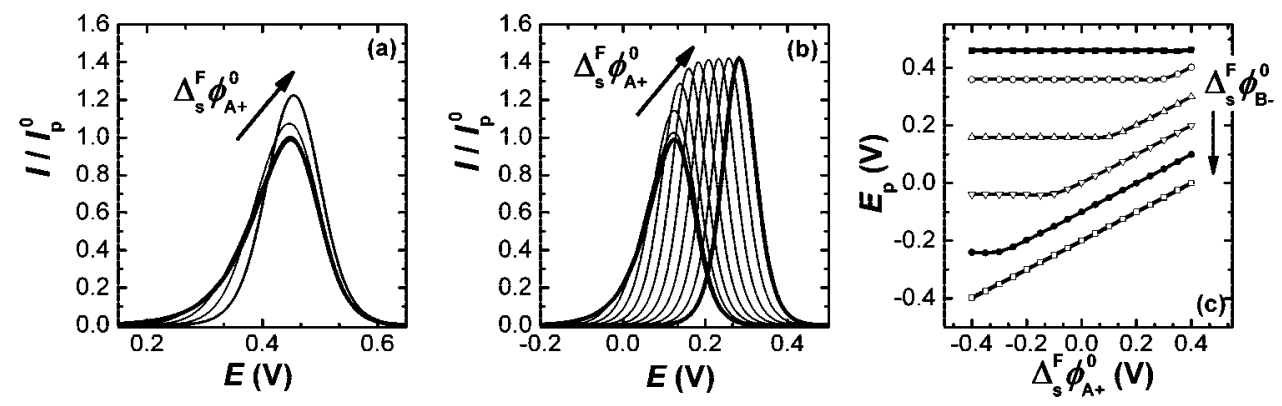

Figure 3. Simulated CVs showing how varying the lipophilicity of the aqueous phase cation $\mathrm{A}+$ can affect both the peak current and position in the $\mathrm{CV}$ for MPC oxidation. $E^{0^{\prime}}=0 \mathrm{~V}$ and standard cation transfer potentials were varied in $50 \mathrm{mV}$ increments from -0.4 to $+0.4 \mathrm{~V}$ for a given counterion (a) $\mathrm{A}^{+} \mathrm{NO}_{3}{ }^{-}$and (b) $\mathrm{A}^{+} \mathrm{PF}_{6}{ }^{-}$. The peak current is normalized by the current in the absence of cation transfer given by eq 14. (c) Plot of peak potential vs $\Delta_{\mathrm{s}}^{\mathrm{F}} \phi_{\mathrm{A}}^{0^{\prime}}$ for a series of anions ranging from very hydrophilic $\left(\Delta_{\mathrm{s}}^{\mathrm{F}} \phi_{\mathrm{B}}^{0^{\prime}}=0.5 \mathrm{~V}\right)$ to very hydrophobic $\left(\Delta_{\mathrm{s}}^{\mathrm{F}} \phi_{\mathrm{B}}^{0^{\prime}}=-0.4 \mathrm{~V}\right)$. Values for $\Delta_{\mathrm{s}}^{\mathrm{F}} \phi_{\mathrm{B}}^{0^{\prime}}$ were $0.5,0.4 .0 .2,0,-0.2,-0.4 \mathrm{~V}$, top to bottom.

increasing $\Delta_{\mathrm{s}}^{\mathrm{F}} \phi_{\mathrm{A}}^{0^{\prime}}$. However, there is no comparable shift in $E_{\mathrm{p}}$ for the range of $\Delta_{\mathrm{S}}^{\mathrm{F}} \phi_{\mathrm{A}}^{0^{\prime}}$ considered when the anion is $\mathrm{NO}_{3}{ }^{-}$ (Figure 3a). This demonstrates how the counterion, here either $\mathrm{PF}_{6}{ }^{-}$or $\mathrm{NO}_{3}{ }^{-}$, determines whether a given cation can partition from the aqueous to the film phase. The hydrophilic $\mathrm{NO}_{3}{ }^{-}$ prevents very lipophilic cations from entering the film at equilibrium, and thus, there are no cations available in the film to be ejected in the coupled ET-IT reaction. In contrast, the relatively hydrophobic $\mathrm{PF}_{6}{ }^{-}$partitions readily into the film when the lipophilicity of the cation is increased and the cation, anion, or combination of both can be coupled ion transfer reaction.

Similar simulations were performed for a range of aqueous phase anions where the lipophilicity was varied from $\Delta_{\mathrm{s}}^{\mathrm{F}} \phi_{\mathrm{B}}^{0^{\prime}}=$ $0.5 \mathrm{~V}$ (very hydrophilic) to $-0.4 \mathrm{~V}$ (very hydrophobic). Values of $E_{\mathrm{p}}$ from each simulated CV were extracted and plotted versus $\Delta_{\mathrm{s}}^{\mathrm{F}} \phi_{\mathrm{A}}^{0^{\prime}}$ for a given anion (Figure 3c). From this figure, it can be readily seen that the dependence of $E_{\mathrm{p}}$ on the cation lipophilicity is determined by the counterion lipophilicity. If the anion is very hydrophilic like $\mathrm{NO}_{3}{ }^{-}$, then $E_{\mathrm{p}}$ is unaffected by cation lipophilicity over a very broad range. In contrast, if the anion is extremely hydrophobic $\left(\mathrm{TPBF}_{20}{ }^{-}\right)$, then $E_{\mathrm{p}}$ varies for all cations regardless of lipophilicity. Anions between these two extremes give an intermediate response where the onset of a shift in $E_{\mathrm{p}}$ is dependent on the relative lipophilicities of both anions and cations. After the onset has been reached, it can be seen that, as cation lipophilicity increases $\left(\Delta_{\mathrm{s}}^{\mathrm{F}} \phi_{\mathrm{A}}^{0^{\prime}}\right.$ increases), $E_{\mathrm{p}}$ shifts positive. This is in contrast to the trend for increasing anion hydrophobicity where for a given cation, $E_{\mathrm{p}}$ shifts negative as the anion becomes increasingly hydrophobic $\left(\Delta_{\mathrm{s}}^{\mathrm{F}} \phi_{\mathrm{B}}^{0^{\prime}}\right.$ decreases). The opposing trends in $E_{\mathrm{p}}$ with increasing anion and cation lipophilicity have their origin in the electroneutrality condition; i.e., cations and anions transfer in opposing directions for MPC oxidative charging. ${ }^{15}$ Thus, for anion injection to the film, hydrophobic anions transfer more readily while for cation ejection from the film, hydrophilic cations transfer more readily. The cation concentration in the film is relevant as it should be greater than or equal to the MPC concentration to be able to fully compensate for the MPC oxidative charging. Experimentally, achieving such a high concentration (here $0.026 \mathrm{M}$ ) simply by ion partitioning is not very likely, and the coupled IT transfer is likely to be mixed anion injection/cation ejection. If we repeat the simulations using lower values for the MPC concentration, the effects of cation lipophilicity on $E_{\mathrm{p}}$ are apparent at lower values of $\Delta_{\mathrm{s}}^{\mathrm{F}} \phi_{\mathrm{A}}^{0^{\prime}}$ (Supporting Information). However, this is an unphysical situation as the MPC concentration is unlikely to vary by more than an order of magnitude regardless of the packing in the film.

It should be remembered that as we are considering oxidative MPC charging, the charge compensating ion transfer reaction is either $\mathrm{B}^{-}(\mathrm{s}) \rightarrow \mathrm{B}^{-}\left(\right.$film) or $\mathrm{A}^{+}($film $) \rightarrow \mathrm{A}^{+}(\mathrm{s})$. In all reported experiments, only the aqueous phase ions and their concentrations have been varied. ${ }^{7-12}$ Neither the type of ions nor their concentration in the film has been directly varied, and thus, these parameters vary only through ion partitioning at equilibrium. In the above simulations and most reports in the literature, ${ }^{7-12}$ changing the aqueous phase concentration shifts $E_{\mathrm{p}}$ to more negative potentials through the anion concentration term in the expression for $E_{\mathrm{p}}$. As the aqueous anion concentration is in vast excess, $\mathrm{B}^{-}(\mathrm{s}) \rightarrow \mathrm{B}^{-}($film $)$is the charge compensating reaction as has been noted in the case of $\mathrm{NH}_{4}{ }^{+} \mathrm{B}^{-}$where $\mathrm{B}^{-}$is $\mathrm{NO}_{3}{ }^{-}$, $\mathrm{BF}_{4}{ }^{-}, \mathrm{ClO}_{4}{ }^{-}, \mathrm{PF}_{6}{ }^{-}$, and $\mathrm{LiTPBF}_{20}{ }^{-}$. However, both the above simulations and data from the literature show that, for a given anion, simply making the cation more lipophilic can influence $E_{\mathrm{p}}{ }^{9}$ An obvious caveat to the above model is that very hydrophilic cations are very unlikely to partition into the film in the first place unless coupled with a very hydrophobic anion. Thus, $\mathrm{NH}_{4} \mathrm{NO}_{3}$ is very unlikely to partition into the film while $\mathrm{LiTPBF}_{20}$ partitions readily.

The influence of a second electrolyte added to the aqueous phase $\mathrm{C}^{+} \mathrm{D}^{-}$was also considered as this is a typical scenario in experimental reports where the concentration of one salt is varied and a second electrolyte is added to maintain the ionic strength of the solution (Supporting Information). From the simulated $\mathrm{CVs}$, the most lipophilic ions determined $E_{\mathrm{p}}$ (Supporting Information). For example, the simulations show that when the aqueous solution contained equal concentrations of $\mathrm{NaNO}_{3}+$ $\mathrm{NaPF}_{6}$, the effect of either $\mathrm{Na}^{+}$or $\mathrm{NO}_{3}{ }^{-}$was not apparent on $E_{\mathrm{p}}$ as $\mathrm{PF}_{6}{ }^{-}$transfer dominated. For a hypothetical solution containing equimolar concentrations of $\mathrm{NaPF}_{6}$ and tetraoctylammonium nitrate $\left(\mathrm{TOANO}_{3}\right)$, the simulated $\mathrm{CV}$ response was identical to that obtained in the presence of just $\mathrm{TOAPF}_{6}$. From the simulations, it can be concluded that the inert electrolyte has to be sufficiently more hydrophilic that the ions under study to exert no influence on $E_{\mathrm{p}}$.

The predictions in Figure $3 \mathrm{c}$ are in excellent agreement with data from the literature with the exception of the $\mathrm{NO}_{3}{ }^{-}$case. ${ }^{9}$ In the literature, ${ }^{9}$ shifts in $E_{\mathrm{p}}$ were noted when this anion was combined with moderately hydrophobic cations like tetraethylammonium and tetramethylammonium $\left(\Delta_{\mathrm{s}}^{\mathrm{F}} \phi_{\mathrm{A}}^{0^{\prime}}\right.$ in the range of -0.27 and $-0.12 \mathrm{~V}$ in Figure 3c) while the simulations predict no change. As it is improbable that $\mathrm{TEANO}_{3}$ would partition extensively into the film due to the hydrophilicity of $\mathrm{NO}_{3}{ }^{-}$, other factors must play a role. $\mathrm{NO}_{3}{ }^{-}$is considerably more hydrophilic than any of the other studied anions, and the effect of cotransported water on the permittivity of the MPC film may be a factor. Increasing the water content of the film would lower the solvation barrier between the phases and facilitate partitioning. ${ }^{17}$ Electrochemical quartz crystal microbalance (EQCM) 
measurements support the concept of cotransported water with hydrophilic ions. ${ }^{7,8}$ It has also been reported that, for the $\mathrm{NH}_{4} \mathrm{NO}_{3}+\mathrm{TEANO}_{3}$ case, $E_{\mathrm{p}}$ shifts positive with increasing mole fraction of $\mathrm{TEANO}_{3}$. This implies that TEA ${ }^{+}$partitioning into the film is such that the concentration in the film is changed significantly and cation ejection controls $E_{\mathrm{p}}$. This is very unlikely within the framework of our model, and further study is required to fully understand this effect.

Experimentally, ion partitioning can also give rise to unexpected experimental features where the films can exhibit considerable memory effects of the electrolyte solutions they have been in contact with. For instance, for a given film used in a series of experiments, we noted that the $\mathrm{CV}$ response in the presence of a given aqueous electrolyte is dependent on which electrolyte it was in contact with last. The response for a fresh film in contact with $\mathrm{NH}_{4} \mathrm{PF}_{6}$ differs significantly if the film has already been in contact with $\mathrm{LiTPBF}_{20}$.

Thus, the measured $E_{\mathrm{p}}$ for the coupled IT-ET reaction depends on the interplay between the MPC concentration, the ion concentration in the film and its lipophilicity, and the aqueous phase anion concentration and lipophilicity. These conclusions concur with those reached for thin film modified electrodes studied extensively by Scholz, Compton, Oldham, and others. ${ }^{15,16,22-24}$

Role of Co-transported Water. In our recent publication, the absence of an onset potential in the presence of very hydrophobic anions is quantitative evidence for the success of the ion-transfer limited model. ${ }^{11}$ This ion transfers into the film at all potentials within the available potential window, and MPC charging peaks were observed throughout the window. The absence of an onset potential was confirmed by EQCM measurements. ${ }^{11}$ Another interesting feature of using this ion is that the peak spacing between successive charging peaks, $\Delta E$, was comparable to that obtained for the same MPCs dispersed in 1,2-dichloroethane. ${ }^{11}$ In contrast, $\Delta E$ for film charging obtained in the presence of the more hydrophilic anions $\left(\mathrm{PF}_{6}{ }^{-}\right.$, $\mathrm{ClO}_{4}{ }^{-}, \mathrm{BF}_{4}{ }^{-}, \mathrm{NO}_{3}{ }^{-}$) was significantly smaller: 150 versus 250 $\mathrm{mV} .^{7-11}$ DCE dispersed MPCs show no such $\Delta E$ dependence on the electrolyte anion at low charge numbers. ${ }^{3,5,25,26}$ This effect can be readily explained when the hydrophobicity of the anion is taken into account. Small relatively hydrophilic anions retain their hydration shell when they are transferred into the film and thus increase the water content of the film. This has been quantified by recent quartz crystal microbalance measurements. ${ }^{7,8}$ The presence of water changes the dielectric properties of the MPC film which in turn affects the MPC capacitance. ${ }^{26,27}$ For dispersed MPCs, the influence of the solvent dielectric constant has been considered and MPC capacitance increases with the solvent dielectric constant, and thus, $\Delta E$ decreases. ${ }^{27}$ Very large hydrophobic anions are weakly solvated by water ${ }^{28}$ and do not cotransport water into the film, and thus, $\Delta E$ is comparable to that reported in dichlorobenzene, a solvent that has a dielectric constant comparable to that for alkanethiols. ${ }^{27}$ Thus, taking ion solvation into account can explain the difference in $\Delta E$ noted for MPC films versus dispersed MPCs.

\section{Conclusions}

The CV response for the oxidative charging of MPC films can be fully described by the ion-limited model. Features that were difficult to rationalize with the ion association model can be quantitatively accounted for, namely, the peak current dependence on film thickness and the influence of cation lipophilicity on the onset potential. The onset potential correlates with the ion transfer potential of the corresponding counterion into the film. Peak current is controlled by the MPC film thickness provided the film is behaving as an ideal thin film. By using a model that simply takes into account the solvation barrier for the transfer of charge compensating counter-ions into the film and considering the effects of ion partitioning on the peak position and current, the charging response of MPC films can be readily quantified.

Acknowledgment. We acknowledge financial support from the University of Helsinki (Development of Nano Sciences/ HENAKOTO, 700036, T.L.), Academy of Finland (V.R. and B.M.Q.), Nederlandse Organisatie voor Wetenschappelijk Onderzoek (NWO/Chemical Sciences, Vidi-grant 700.56.423, P.L.).

Supporting Information Available: Additional experimental data (Langmuir isotherm, TEM image) and additional simulations. This material is available free of charge via the Internet at http://pubs.acs.org.

\section{References and Notes}

(1) Daniel, M. C.; Astruc, D. Chem. Rev. 2004, 104, 293.

(2) Templeton, A. C.; Wuelfing, W. P.; Murray, R. W. Acc. Chem. Res. 2000, 33, 27.

(3) Quinn, B. M.; Liljeroth, P.; Ruiz, V.; Laaksonen, T.; Kontturi, K. J. Am. Chem. Soc. 2003, 125, 6644.

(4) Miles, D. T.; Murray, R. W. Anal. Chem. 2003, 75, 1251.

(5) Hicks, J. F.; Miles, D. T.; Murray, R. W. J. Am. Chem. Soc. 2002, $124,13322$.

(6) Chen, S. W.; Ingram, R. S.; Hostetler, M. J.; Pietron, J. J.; Murray, R. W.; Schaaff, T. G.; Khoury, J. T.; Alvarez, M. M.; Whetten, R. L. Science 1998, 280, 2098

(7) Ruiz, V.; Colina, A.; Heras, M. A.; Lopez-Palacios, J. J. Phys. Chem. C 2007, 111, 4277.

(8) Deng, F.; Chen, S. Langmuir 2007, 23, 936.

(9) Chen, S.; Pei, R. J. Am. Chem. Soc. 2001, 123, 10607.

(10) Chen, S. J. Am. Chem. Soc. 2000, 122, 7420.

(11) Laaksonen, T.; Ruiz, V.; Murtomäki, L.; Quinn, B. M. J. Am. Chem. Soc. 2007, 129, 7732.

(12) Chen, S.; Pei, R.; Zhao, T.; Dyer, D. J. J. Phys. Chem. B 2002, 106, 1903.

(13) Brennan, J. L.; Branham, M. R.; Hicks, J. F.; Osisek, A. J.; Donkers, R. L.; Georganopoulou, D. G.; Murray, R. W. Anal. Chem. 2004, 76, 5611.

(14) Jhaveri, S. D.; Lowy, D. A.; Foos, E. E.; Snow, A. W.; Ancona,

M. G.; Tender, L. M. Chem. Commun. 2002, 1544.

(15) Scholz, F.; Gulaboski, R. ChemPhysChem. 2005, 6, 16.

(16) Banks, C. E.; Davies, T. J.; Evans, R. G.; Hignett, G.; Wain, A. J.; Lawrence, N. S.; Wadhawan, J. D.; Marken, F.; Compton, R. G. Phys. Chem. Chem. Phys. 2003, 5, 4053.

(17) Liquid-Liquid Interfaces, Theory and Methods; Volkov, A. G., Deamer, D. W., Eds.; CRC Press: Boca Raton, FL, 1996.

(18) Bard, A. J.; Faulkner, L. R. Electrochemical Methods, Fundamentals and Applications, 2nd ed.; John Wiley and Sons Inc.: New York, 2001.

(19) Hicks, J. F.; Zamborini, F. P.; Osisek, A. J.; Murray, R. W. J. Am. Chem. Soc. 2001, 123, 7048.

(20) Hundhammer, B.; Muller, C.; Solomon, T.; Alemu, H.; Hassen, H. J. Electroanal. Chem. 1991, 319, 125.

(21) Kakiuchi, T. Anal. Chem. 1996, 68, 3658.

(22) Bobacka, J.; Gao, Z.; Ivaska, A.; Lewenstam, A. J. Electroanal. Chem. 1994, 368, 33.

(23) Lewenstam, A.; Bobacka, J.; Ivaska, A. J. Electroanal. Chem. 1994, $368,23$.

(24) Myland, J. C.; Oldham, K. B. Electrochem. Commun. 2000, 2, 541.

(25) Laaksonen, T.; Pelliniemi, O.; Quinn, B. M. J. Am. Chem. Soc. 2006, $128,14341$.

(26) Garcia-Morales, V.; Mafe, S. J. Phys. Chem. C 2007, 111, 7242.

(27) Su, B.; Zhang, M.; Shao, Y.; Girault, H. H. J. Phys. Chem. B 2006, $110,21460$.

(28) Krossing, I.; Raabe, I. Angew. Chem. 2004, 43, 2066.

JP8048108 